\title{
CdSe QDs reinforced poly(1, 8 diaminonaphthalene) (PDAN) offers improved thermal and AC conductivity properties
}

\author{
Rashmi Singh $^{1} \cdot$ Anil Kumar Bajpai ${ }^{2} \cdot$ Ashwani Kumar Shrivastava $^{1}$
}

(c) Springer Nature Switzerland AG 2019

\begin{abstract}
Conducting polymers have been identified as materials with low conductivity and poor environmental and thermal stabilities. In order to improve the conductivity and stability properties, reinforcement of semiconducting nanoparticles into polymer matrix has been successfully attempted. In the present study, CdSe quantum dots (QDs) were reinforced into poly (1, 8 diaminonaphthalene) (PDAN) matrix to design PDAN-QDs nanocomposites which were characterized by transmission electron microscopy (TEM) technique. The results showed that the monomer DAN showed spherical morphology while upon polymerization its polymer PDAN acquired chain type morphology having size less than $1 \mu \mathrm{m}$. The TEM images of PDAN/CdSe composite showed that the QDs had size in the range of 4-7 nm which get embedded into the polymer matrix during polymerization. X-ray diffraction pattern of PDAN/CdSe nanocomposites revealed the presence of cubic as well as hexagonal phases. The thermal degradation and electrical conduction of this composite were found to be enhanced as compared to those of the native PDAN.
\end{abstract}

\section{Graphic abstract}

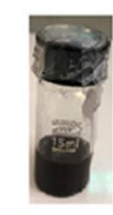

Ammonium Persulphate

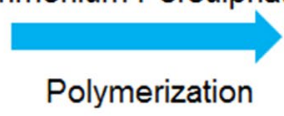

DAN Solution

Polymerization

DAN Solution
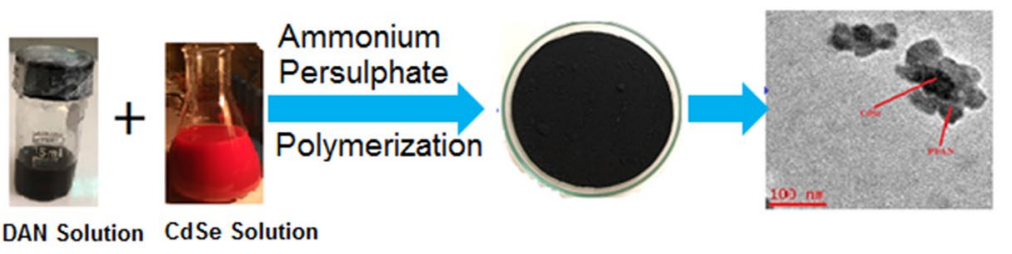

PDAN/CdSe Nanocomposite

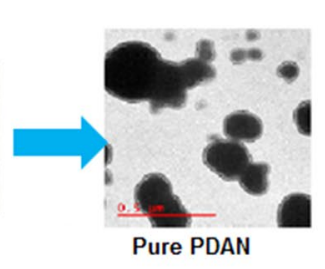

PDAN

PDAN/CdSe Nanocomposite

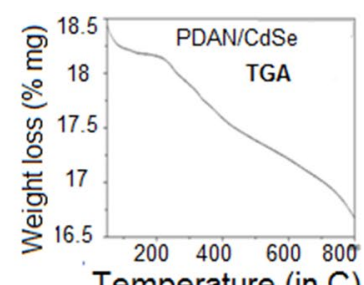

Temperature (in C)

Temp.Vs.Weight Loss

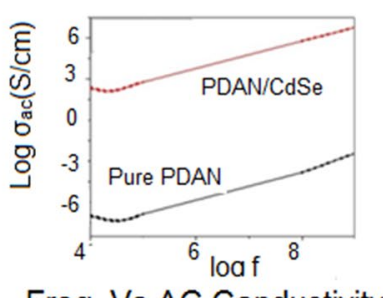

Freq. Vs AC Conductivity

Keywords Polymer · Nanocomposites · Thermal stability · Nanomaterial · Quantum dots · Conductivity

Rashmi Singh, rash.pinki@gmail.com | ${ }^{3}$ School of Studies in Physics, Jiwaji University, Gwalior 474011, India. ${ }^{2}$ Bose Memorial Research Laboratory, Department of Chemistry, Govt. Autonomous Science College, Jabalpur 482002, India.

SN Applied Sciences (2019) 1:815 | https://doi.org/10.1007/s42452-019-0835-3

Received: 23 April 2019 / Accepted: 24 June 2019 / Published online: 1 July 2019 


\section{Introduction}

The energy consumption has been increasing uninterruptedly and in last few decades it has become havoc. The increasing world population has necessitated exploring and designing superior energy storage and transformation devices to fulfill basic needs of human life [1]. Conducting polymers and their hybrid composites with semiconductor materials have revolutionized the scenario of energy storage devices due to their inherent properties like light weight, improved conductivity, greater thermal stability etc. The devices fabricated from these materials can be used in aerospace structures, conductive coatings, electromagnetic shielding and other thermal and electrical devices $[2,3]$.

Among various available conducting polymers, poly(diamino naphthalene) (PDAN) is a known conjugated polymer having electron donating group which enables it to show chelating and reduction properties $[4,5]$. These electron donating groups, imine $(-\mathrm{N}=\mathrm{C})$, amine $(-\mathrm{NH}-\mathrm{C})$ and free amine $\left(-\mathrm{NH}_{2}\right)$ are linked to naphthalene rings. PDAN is a p-type semiconductor which is more stable than other conjugated polymers. The chemistry of PDAN has been discussed by Li et al. [6]. The PDAN finds applications in the fabrication of Schottky diode also [7]. The electro catalytic reduction of oxygen molecules using PDAN has also been reported by Park et al. The PDAN is also used to remove $\mathrm{Cr}$ ions from sewage waste [6]. Majority of researcher have used electrochemical or chemical oxidative polymerization method for the preparation of $\operatorname{PDAN}[7,8]$.

Polymer nanocomposites are intimately compatible polymer systems in which at least one of the components falls into the nano-range dimension and homogenously dispersed into the polymer matrix. These nanocomposites are quite advantageous in the sense that they offer much improved properties in comparison to those of their counter parts and, thus, results in value addition to the end material. The ultimate properties of nanocomposites greatly depend on the factors such as chemical nature of the polymer matrix and the filler being dispersed, particles size and size distribution of the filler, interactions between the polymer and filler, state of dispersion of the filler, molecular weight of the polymer, and method of fabrication of the nanocomposite. By precise manipulation of these factors one can desirably achieve the polymer nanocomposite of required properties for intended end applications [9-12].

Quantum confinement plays a prominent role in nanoscience and nanotechnology. If the size of CdSe nanoparticles is of the order of 1-10 nm, they show quantum confinement effect. When the $\mathrm{CdSe}$ particle size approaches to Bohr radius, it results in increasing exciton transition energy and the absorption spectra show a blue shift. The quantum confinement also causes change in the atomic structure of $\mathrm{CdSe}$ nanoparticles. This confinement causes the electron to feel the presence of the particle boundaries, which in turn responds to the change in particle size by adjusting their energies. This feature of (CdSe) depends upon its size, shape and structure $[13,14]$. The thermal behavior and stability of CdSe quantum dots (QDs) are important parameters to decide their application in fabrication of devices. The electronic devices fabricated using CdSe QDs are thermally more stable than any other organic polymers [15-20].

For designing protective and sustainable electronic devices of polymer nanocomposites, thermal stability leads a key role. If composite is heated at high temperature (higher than melting point) it can melt or degrade which can result in the internal short circuit. So for designing protective electronic devices, thermal stability plays a crucial role [21]. Thermal gravimetric analysis (TGA) provides thermal decomposition data and determines thermal stability of the materials. It also measures the weight gain or loss by the sample when heated in an inert atmosphere with a pre-determined heating rate. When polymer nanocomposites are heated over a particular range of temperature, the information about their thermal stability at different temperatures can be drawn [22]. TGA of sample under investigation depends on temperature, pressure and particle size of the filler material. Thermal analysis of conducting polymer and its nanocomposites are very important for device fabrications and also in many technological applications [23].

The main aim of the present work is to improve structural, thermal and electric properties of CdSe QDs reinforced PDAN by using chemical oxidative polymerization method. Earlier people have studied the polymer and semiconductor material independently. The literature related to the composite of PDAN and CdSe lacks in their thorough study. The choice of semiconductor nanoparticles in composite has further modified the material having enhanced properties. The present method provides large yield in less time and is also cost effective. The present polymer nanocomposites are light weight, high electrically conducting and thermally stable. Thus, light weight energy storage devices, sensors and thermal devices can be designed which can be used in space shuttles, aeronautic engineering technology, polymer electronic industries, and also in various prospective applications.

\section{Methodology}

\subsection{Materials}

The chemicals used during the course of present investigation were cadmium acetate dihydrate (98\%), selenium 
(Se) (metal) powder (99\%), thiourea (99\%), 1, 8-diaminonaphthalene (DAN) (96\%), ammonium persulphate (APS) $\left(\left(\mathrm{NH}_{4}\right)_{2} \mathrm{~S}_{2} \mathrm{O}_{8}\right)(98 \%)$, triethanolamine (TEA) (97\%) and nitric acid $\left(\mathrm{HNO}_{3}\right)$ (71\%). They were obtained from HIMEDIA, Thermo Fisher Scientific India Pvt. Ltd. and Central Drug House, New Delhi, India. Acetonitrile (99\%) and de-ionized water were used as solvents.

\subsection{Polymerization of 1, 8-diaminonaphthalene (DAN)}

The solution of monomer (DAN) was prepared by dissolving $0.63 \mathrm{M}$ of DAN in $40 \mathrm{~mL}$ acetonitrile. To this solution, $0.5 \mathrm{M}$ aqueous solution of APS was added dropwise and thereafter the resulting solution was ultrasonicated at $20 \mathrm{kHz}$. After ultrasonification, the solution was kept at temperature $\left(5^{\circ} \mathrm{C}\right)$ for a period of $10 \mathrm{~h}$ to complete the polymerization reaction. The viscous solution so obtained was filtered and washed with distilled water and acetone to remove unreacted chemicals and impurities. The washed material was dried at room temperature for 3 days. In this method $95 \%$ yield was obtained. The material so obtained was black colored powder of PDAN which was stored in airtight polyethylene bags for subsequent studies.

\subsubsection{Mechanism of polymerization of DAN}

$\mathrm{S}_{2} \mathrm{O}_{8}^{2-} \rightarrow 2 \mathrm{SO}_{4}^{--}\left(\mathrm{R}^{\cdot}\right)$

$\mathrm{R}+\mathrm{DAN} \rightarrow \mathrm{R}-\mathrm{DAN}$

$\mathrm{R}-\mathrm{DAN}+\mathrm{n}$ DAN $\rightarrow \mathrm{R}(\mathrm{DAN})_{(\mathrm{n}+1)}^{{ }_{(1)}}$

$\mathrm{R}(\mathrm{DAN})_{(\mathrm{n}+1)}+\mathrm{R}(\mathrm{DAN})_{(\mathrm{m}+1)} \rightarrow \mathrm{R}(\mathrm{DAN})(\mathrm{n}+1)-(\mathrm{DAN})(\mathrm{m}+1)$

According to Eq. (1) of the proposed mechanism, the persulphate ion dissociates to give two sulphate ion radicals ( $R$ ) which reacts with the monomer DAN to give monomer radical (Eq. 2). The as- produced monomer radical reacts with ' $\mathbf{n}$ ' number of DAN molecules and produces DAN macroradicals (Eq. 3). Such two macroradicals of DAN combine mutually and forms a macromolecule of DAN (Eq. 4). This is how the proposed mechanism gives the formation of PDAN.

\subsection{Synthesis of CdSe nanoparticles}

CdSe nanoparticles were obtained using chemical coprecipitation method. In a typical experiment, $2.53 \mathrm{M}$ of Se metal powder was taken in a flask and $3 \mathrm{~mL} \mathrm{HNO}_{3}$ was added to it till it dissolves completely. Thereafter, $20 \mathrm{~mL}$ double distilled water was added to this solution. The solution was continuously stirred and refluxed at $80^{\circ} \mathrm{C}$ for
$12 \mathrm{~h}$. After refluxing was over, the mixture was filtered and kept in dark. This solution was named as 'A'. Now, $2 \mathrm{M}$ thiourea was dissolved in $10 \mathrm{~mL}$ of di-ionized water and $1.5 \mathrm{M}$ cadmium acetate was dissolved in $20 \mathrm{~mL}$ di-ionized water, separately. Both the solutions were mixed drop by drop to get solution B. Now, $20 \mathrm{~mL}$ of above prepared 'A' solution was taken out and mixed with ' $\mathrm{B}$ ' solution. The color of the resulting mixture changed to milky-yellow-orange and finally turned to dark red. When we add thiourea in cadmium acetate solution, molecules of thiourea chelated with $\mathrm{Cd}^{2+}$ ion thus prohibited $\mathrm{S}^{2-}$ ions to form $\mathrm{CdS}$. Afterward, $\mathrm{Cd}^{2+}$ ions react with $\mathrm{Se}^{2-}$ ions and lead to the formation of $\mathrm{CdSe}$ nanoparticles. Now a highly stable $\mathrm{CdSe}$ nanoparticles were obtained that might be due to the use of thiourea as a stabilizing agent [24]. Now $3 \mathrm{~mL}$ of TEA was added to this solution and $\mathrm{pH}$ of solution was maintained at 12 by adding $25 \%$ aqueous ammonia. At this $\mathrm{pH}$ the material gets precipitate. This precipitate was filtered and centrifuged at $9000 \mathrm{rpm}$ for $15 \mathrm{~min}$. After centrifuging, the precipitate particles were again washed and then dried in a hot air oven at $200{ }^{\circ} \mathrm{C}$ for $6 \mathrm{~h}$. On drying the powdered $\mathrm{CdSe}(\mathrm{S})$ nanoparticles were obtained.

$\mathrm{Se}($ Metal $)+2 \mathrm{HNO}_{3} \rightarrow \mathrm{H}_{2} \mathrm{SeO}_{3}+\mathrm{NO}_{2}+\mathrm{NO}$

$\mathrm{H}_{2} \mathrm{SeO}_{3} \rightarrow\left(\mathrm{SeO}_{3}\right)^{-2}+2 \mathrm{H}^{+}$

$\left(\mathrm{SeO}_{3}\right)^{-2}+6 \mathrm{H}^{+}+6 \mathrm{e}^{-} \rightarrow \mathrm{Se}^{2-}+3 \mathrm{H}_{2} \mathrm{O}$

(due to moderate oxidizing nature)

$\mathrm{Cd}\left(\mathrm{CH}_{3} \mathrm{COO}\right)_{2} \rightarrow \mathrm{Cd}^{2+}+2 \mathrm{CH}_{3} \mathrm{COO}^{-}$

$\mathrm{Cd}^{2+}+\mathrm{Se}^{2-} \rightarrow \mathrm{CdSe}$

Thus, these reactions gives the formation of $\mathrm{CdSe}$ nanoparticles (Figs. 1, and 2).

\subsection{Synthesis of PDAN/CdSe nanocomposite}

The effective and low cost chemical oxidative polymerization method was used to prepare PDAN/CdSe(S) polymer-semiconductor nanocomposites. In this method, $0.19 \mathrm{M}$ of DAN was mixed with $40 \mathrm{~mL}$ acetonitrile and the solution was stirred for $20 \mathrm{~min}$. CdSe nanoparticles were then added to this solution. In order to have a homogenous solution of both the DAN and CdSe, the mixture was ultrasonicated at $20 \mathrm{kHz}$. To initiate polymerization, $0.19 \mathrm{M}$ of APS, an oxidizing agent, was added to this solution. The polymerization was allowed to proceed at $5{ }^{\circ} \mathrm{C}$ for $24 \mathrm{~h}$. When the polymerization was complete, the material was separated out and preserved for $48 \mathrm{~h}$ at room temperature. The $\%$ yield produced was $90-95 \%$. 


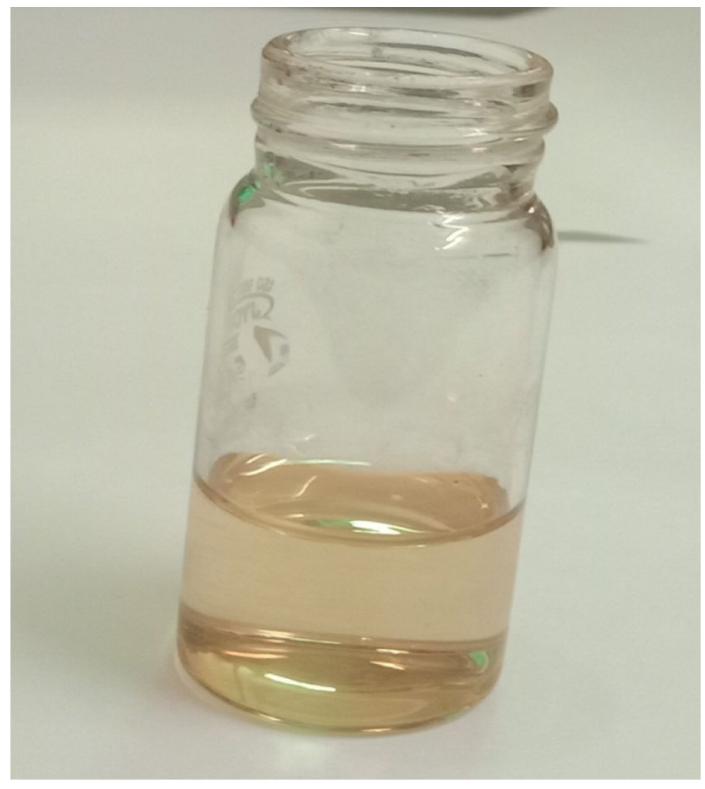

Fig. 1 Aqueous solution containing selenium metal

Finally a black color powder of PDAN/CdSe nanocomposites was obtained which was stored in an air-tight container for further studies.

\subsection{Material characterization}

The X-ray diffraction (XRD) measurements of the so prepared material was recorded on Bruker D8-Discover system using $\mathrm{Cu}-\mathrm{K}_{\mathbf{a}}$ radiation $(\lambda=1.5406 \AA$ ). TEM studies were conducted using Transmission Electron Microscope [JEOL-1230]. Thermal analysis was carried out using Shimadzu's TGA-50 Series Thermo Gravimetric Analyzer. AC conductivity measurements were carried out on model $\mu$-AUTOLAB Type III (Eco-chemie) Potentiostat-galvanostat.

\section{Results and discussion}

\subsection{X-ray diffraction analysis (XRD)}

The XRD pattern of native PDAN, native CdSe and PDAN/ CdSe nanocomposites are shown in Fig. 3. The spectra of PDAN show one broad diffraction peak (110) at $2 \theta=26^{\circ}$ suggesting its amorphous nature. The characteristic peaks of CdSe are observed at $2 \theta$ values of $25^{\circ}, 42^{\circ}, 45^{\circ}$ and $49^{\circ}$ which are identified as $(111,220,103)$ and $(311)$, respectively. Peaks $(111,220$, and 311$)$ are characteristic peaks of the cubic phase, whereas the peak (103) refers to the

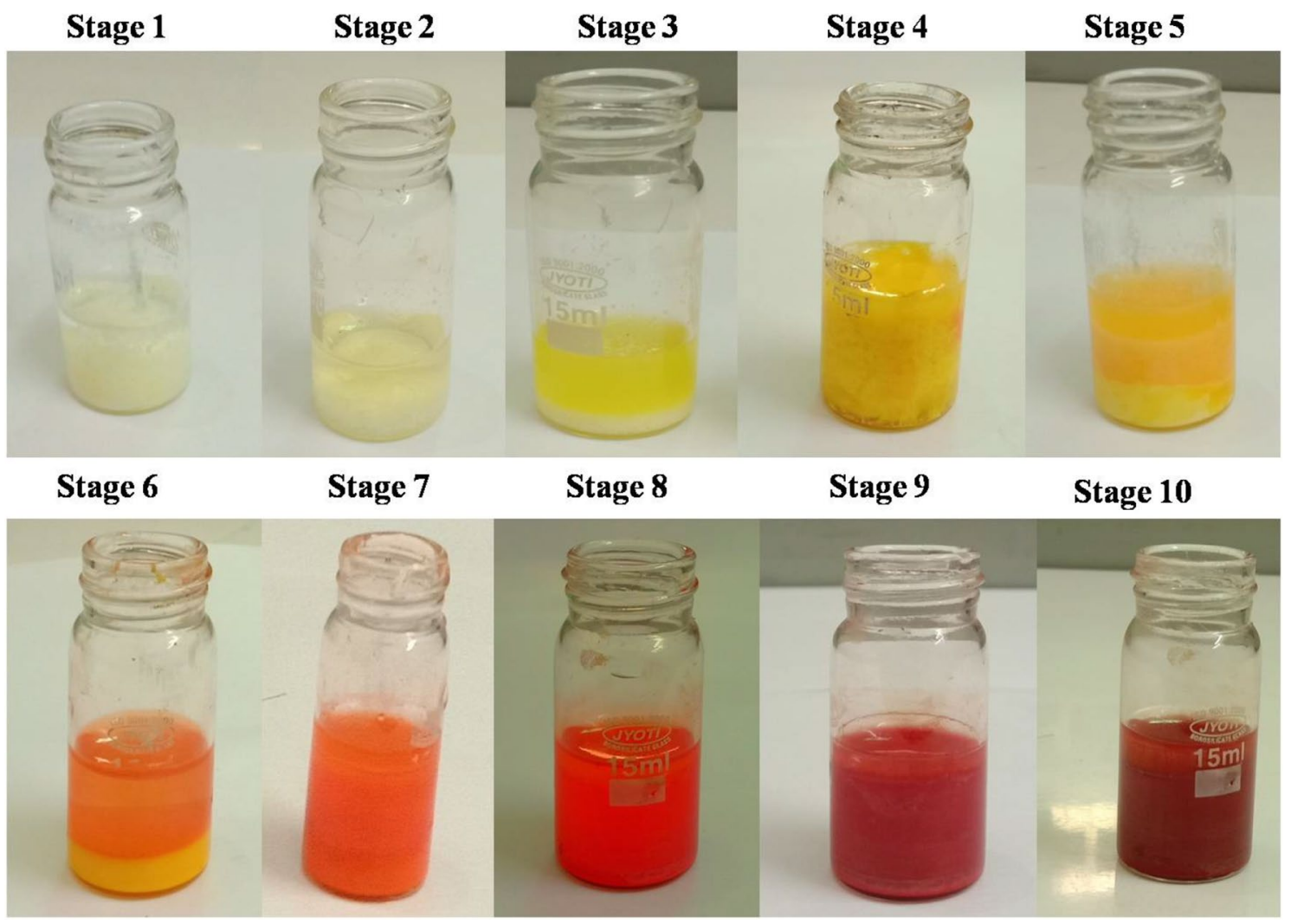

Fig. 2 Different stages during synthesis of CdSe nanoparticles 


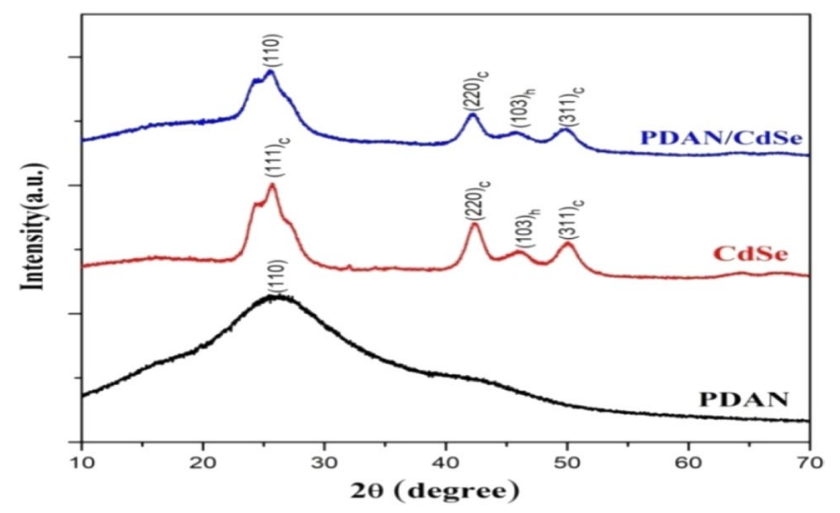

Fig. 3 XRD spectra of native PDAN, CdSe and PDAN/CdSe nanocomposites

hexagonal phase. The XRD spectra of PDAN/CdSe shows one (111) prominent peak at $2 \theta=25^{\circ}$, which is same as that of the CdSe and refers to cubic phase. The (111) peak of CdSe appears to get overlapped with (110) of pure PDAN in the XRD spectra [8]. The additional peaks $(111,220$, and 311) of PDAN/CdSe nanocomposites are characteristic of cubic and (103) hexagonal phases. These peaks are consistent with those of JCPDS card No. 77-2307 and 65-2891. The lattice constants of pure CdSe and PDAN/CdSe nanocomposites were found to be $6.03 \AA$ and $5 \AA$, respectively which match well with the JCPDS card no. 19-0191 [25].

The crystallite size of conducting polymer and its nanocomposites can be calculated using Debye Scherer's formula $[26,27]$

$D=\frac{K \lambda}{\beta \cos \theta}$

$D$ being the crystallite size; $K(0.9)$ : Scherer's constant; $\lambda$ (1.5406 $\AA$ ): $X$-ray wavelength; $\theta$ : Bragg's angle and $\beta$ is the full width at half maximum in radian. The crystallite size of pure $\mathrm{CdSe}$, as calculated from XRD spectra is $3.0 \mathrm{~nm}$. When $\mathrm{CdSe}$ is incorporated in to the PDAN matrix viz. PDAN/CdSe the crystallite size changes to $4 \mathrm{~nm}$. The XRD spectra further reveal that the incorporation of $\mathrm{CdSe}$ into organic polymer matrix decreases the amorphous nature of PDAN [28, 29]. It is also worth to mention here that the decrease in the diffracted X-ray intensity and broadening of halo peak indicates the decrease of amorphous region content, might be due to the interaction between the PDAN matrix and QDs. The detailed parameters of XRD are shown in Table 1.

\subsection{Transmission electron microscopy (TEM) analysis}

TEM analysis shows internal morphology of PDAN and its nanocomposites (PDAN/CdSe). Figure 4a shows particles of DAN, which are perfectly spherical with a diameter of
Table 1 XRD data of pure PDAN, pure CdSe and PDAN/CdSe nanocomposites

\begin{tabular}{llll}
\hline Parameters & Pure PDAN & Pure CdSe & $\begin{array}{l}\text { PDAN/CdSe } \\
\text { nanocom- } \\
\text { posites }\end{array}$ \\
\hline${\text { Prominent peak at } 2 \theta\left(\text { in }^{\circ}\right)}^{\circ}$ & $26.32^{\circ}$ & $25.54^{\circ}$ & $25.15^{\circ}$ \\
Intensity (a.u.) & 5944 & 4782 & 4284 \\
FWHM ( $\beta$ ) (in $\left.{ }^{\circ}\right)$ & 10.99 & 2.783 & 3.9 \\
Miller indices (h k l) & $(110)$ & $(111)$ & $(110)$ \\
\hline
\end{tabular}

$70 \mathrm{~nm}$. On polymerization, the DAN particles join together and make a long chain of PDAN which are clearly visible in Fig. 4b. Bhatt et al. [30] have also reported similar spherical morphology of PDAN particles in their work.

Figure 5a, shows the particle morphology in PDAN/ CdSe nanocomposites, where large number of CdSe nanoparticles having size 4-7 $\mathrm{nm}$ are surrounded by PDAN matrix. Figure $5 \mathrm{~b}$ reveals that agglomerated $\mathrm{CdSe}$ nanoparticles are centered and surrounded by PDAN matrix.

The diameter, volume, and surface area of PDAN particles and surface area of CdSe particles in PDAN/CdSe nanocomposites are summarized in Table 2.

\subsection{Thermal gravimetric analysis (TGA)}

TGA study was performed in the temperature range, $30-400^{\circ} \mathrm{C}$ for PDAN and $30-800^{\circ} \mathrm{C}$ for PDAN/CdSe nanocomposites, respectively. The TGA plot of pure PDAN and its nanocomposites are shown in Fig. 6. The TGA plot of native PDAN shows two steps degradation. The first step degradation in the PDAN matrix occurs from 70 to $100^{\circ} \mathrm{C}$ and the corresponding weight loss occurring is about $3.2 \%$ that may be due to the evaporation of water molecules and un-reacted monomer from PDAN matrix. The second step degradation occurs over the temperature range of $200-330{ }^{\circ} \mathrm{C}$, where $8.12 \%$ weight loss is observed. The second step weight loss might be due to breakdown of polymer backbone, polymer chain scission, intermolecular and intramolecular cyclization and condensation, structural degradation, and possibility of leaving high amount of residual. It is worth to mention here that the breakdown of polymer backbone normally results in production of free radicals which diffuse into polymer chains and cause scission of polymer chains. All these factors are collectively responsible for thermal degradation of polymer [21]. Similar two step degradation process and thermal stability in polypropylene (PP) and poly lactic acid (PLA) polymer matrix have also been reported by Mofokeng et al. [31], Huda et al. [32] and Yesappa et al. [33].

The thermogram of PDAN/CdSe nanocomposites also shows two steps decay. The first step decay occurs over the temperature range $150-250^{\circ} \mathrm{C}$ and the second step decay 

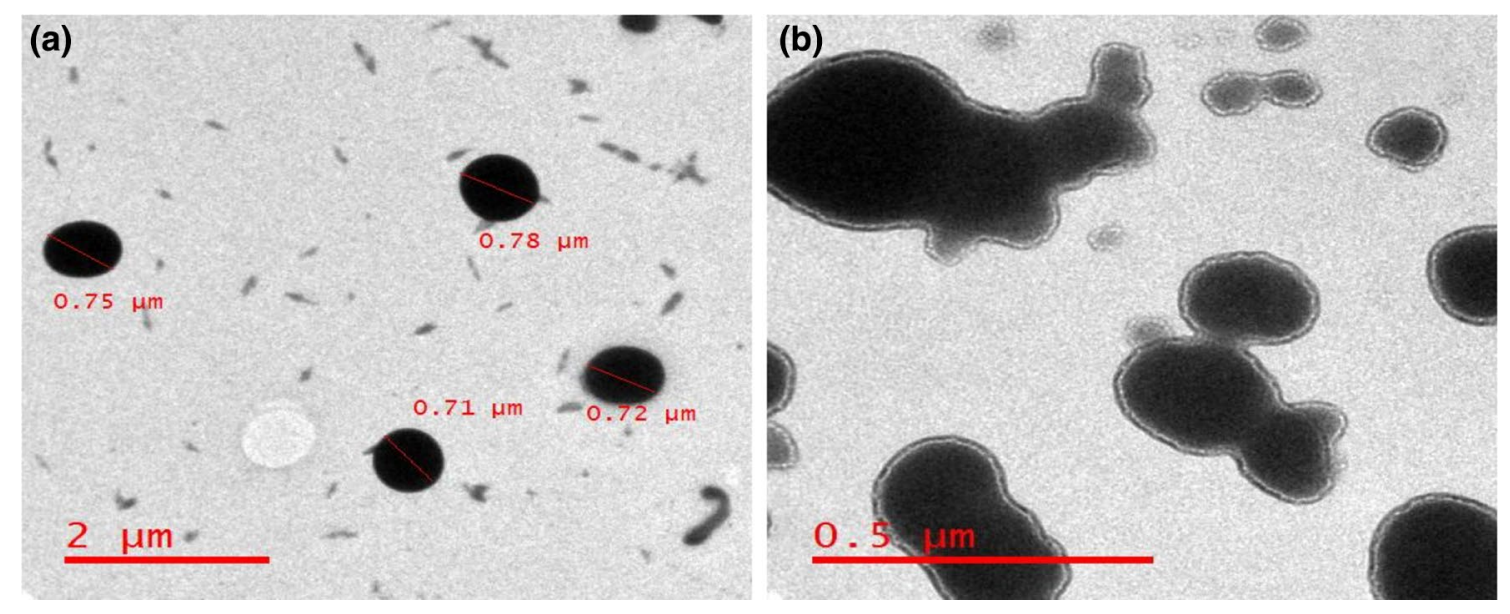

Fig. 4 TEM images of pure PDAN a $2 \times 10^{-6}$ magnification and, b $5 \times 10^{-6}$ magnifications
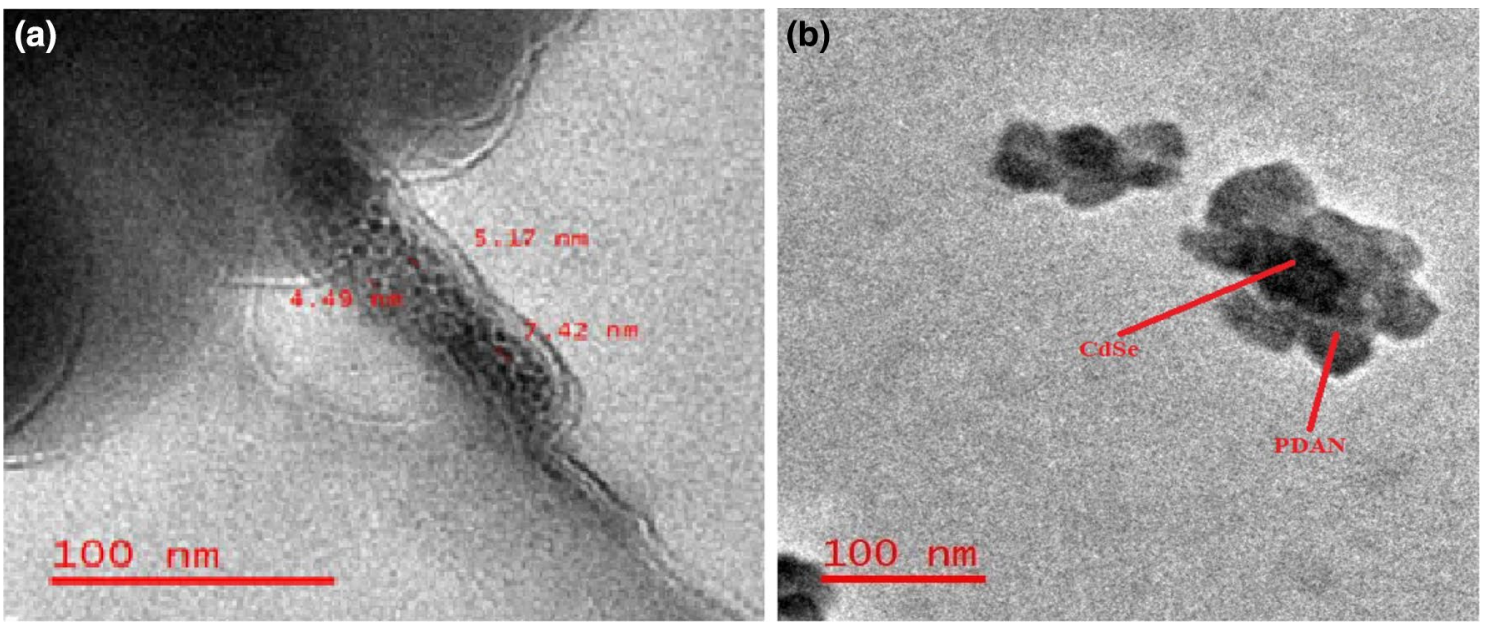

Fig. 5 a, b TEM images of PDAN/CdSe nanocomposites in $1 \times 10^{-7}$ magnification range

Table 2 Structural and morphological parameters for pure PDAN and PDAN /CdSe nanocomposites

\begin{tabular}{ll}
\hline Parameters & Pure PDAN \\
\hline $\begin{array}{ll}\text { (a) For pure PDAN } \\
\text { Diameter of particles }(\mathrm{m})\end{array}$ & $0.74 \times 10^{-6}$ \\
Volume of particles $\left(\mathrm{m}^{3}\right)$ & $0.209 \times 10^{-6}$ \\
Surface area of particles $\left(\mathrm{m}^{2}\right)$ & $1.71 \times 10^{-6}$ \\
\hline Parameters & PDAN/CdSe \\
& nanocomposites \\
\hline (b) For PDAN/CdSe nanocomposites & \\
Diameter of CdSe particles $(\mathrm{m})$ & $20.83 \times 10^{-9}$ \\
Volume of CdSe particles $\left(\mathrm{m}^{3}\right)$ & $3.78 \times 10^{-5}$ \\
Surface area of CdSe particles $\left(\mathrm{m}^{2}\right)$ & $5.44 \times 10^{-6}$ \\
\hline
\end{tabular}

takes place thereafter upto $480^{\circ} \mathrm{C}$. The loss in weight is $2.36 \%$ in the first step decay whereas in the second step the decay is about $6.65 \%$. The first step degradation is due to the loss of capping agent, moisture, un-reacted monomers and other impurities in the synthesis of CdSe. The second step degradation is caused by the decomposition of polymer backbone [22, 31]. Verma et al. [15] have also investigated and reported similar degradation and thermal stability of CdSe quantum dots (QDs). Yesappa and coworkers have also studied the thermal behavior of $\mathrm{PANI}-\mathrm{LiClO}_{4}$ nanocomposites. Abusalemhamadnabirquidri et al. [2] and Yesappa et al. [33] have also reported two step degradation processes while studying thermal properties of PANI/Lead oxide nanocomposites. In CdSe reinforced PDAN nanocomposites, the weight loss are decreased in comparison to pure PDAN. This causes an increase in ductile or plasticity when CdSe is reinforced with PDAN matrix [21]. The obtained greater thermal stability of CdSe/PDAN nanocomposite as compared to pure PDAN may be attributed to the fact that the reinforced $\mathrm{CdSe}$

\section{SN Applied Sciences}



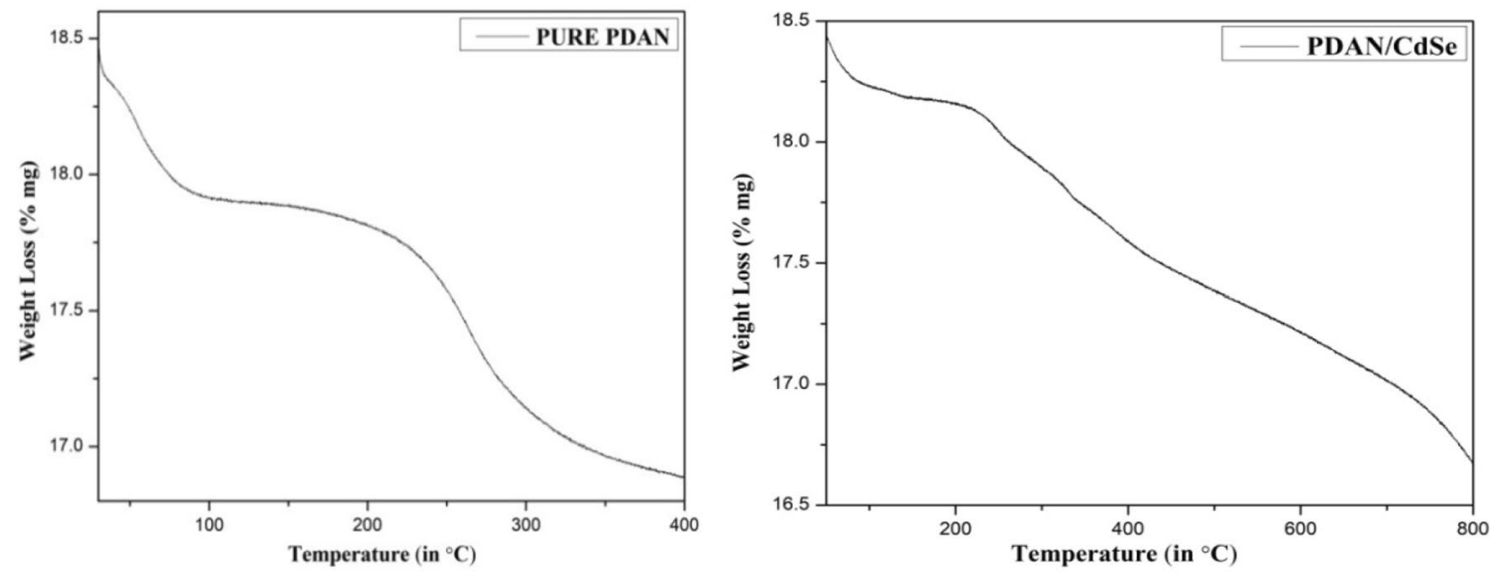

Fig. 6 TGA plots of PDAN and PDAN/CdSe nanocomposites

nanoparticles interact with the macromolecular chains of PDAN and restrains their mobility during the thermal treatment. Thus, the reduced movements of PDAN chains in the nanocomposites results in greater dimensional stability to the material and enable it more thermally stable. Similar type of results has also been reported elsewhere [34]. Since the degradation in composites takes place at higher temperatures, hence it is implied that PDAN/CdSe nanocomposites are more stable than pure PDAN. During the degradation of the polymer chains in CdSe/PDAN nanocomposites, free radicals are generated which in turn enhance the stability of PDAN/CdSe nanocomposites.

The basic mechanism involved in the increase in thermal stability of the material depends upon its capability of dissipating the heat generated. The nanoparticles play an important role in achieving this property as they can easily diffuse and get agglomerated within or over the surface of the host polymer material (PDAN). In the present study, the CdSe nanoparticles diffuse within the polymer material and get agglomerated in the PDAN matrix. The process of agglomeration is clear from the TEM results shown in Fig. 5. These agglomerated centers of nanoparticles act as good radiator of thermal energy which, in turn, increases the thermal stability of the material. The enhanced thermal stability through agglomerated gold nanoparticles in gamma-irradiated polyethylene (PEO)/polyvinylpyrrolidone (PVP) was reported by Abdelghany et al. [21].

Ansari and Keivani [35] and Wei and Hsueh [36] have reported three steps degradation and thermal stability of polyaniline (PANI). Thermal stability of organic material, PDAN in composite depends upon inorganic material like CdSe. In nanocomposites CdSe is attached to PDAN matrix. These results reveal that thermally stable polymer nanocomposites can be used in different potential technological applications such as batteries, optoelectronic devices, charge storage devices etc [37] (Table 3).
Table 3 Data showing the thermal degradation parameters deduced from TGA study

\begin{tabular}{lll}
\hline Thermal parameters & PDAN & $\begin{array}{l}\text { PDAN/CdSe } \\
\text { nanocompos- } \\
\text { ites }\end{array}$ \\
\hline First degradation temperature $\left(\right.$ in $\left.^{\circ} \mathrm{C}\right)$ & $70-100$ & $150-250$ \\
Second degradation temperature $\left(\right.$ in $\left.^{\circ} \mathrm{C}\right)$ & $200-330$ & 480 \\
First weight loss (in \%) & 3.20 & 2.36 \\
Second weight loss (in \%) & 8.12 & 6.65 \\
\hline
\end{tabular}

\subsection{Ac conductivity measurements}

The AC conductivity of pure PDAN and PDAN/CdSe nanocomposites over the pulse repetition frequency range, few $\mathrm{Hz}$ to $100 \mathrm{MHz}$ are shown in Fig. 7. The operating voltage for pure PDAN was kept at $0.20 \mathrm{~V}$ and for PDAN/CdSe nanocomposites, $0.176 \mathrm{~V}$. The plot shows two regions. The first region is lower frequency region which is frequency independent. In this region, the conductivity remains constant in spite of the change in frequency. This represents DC component of the conductivity. The second region is higher frequency region where conductivity is frequency dependant. In this region, the conductivity increases on increasing the applied frequency for native PDAN and PDAN/CdSe nanocomposites. Transition from DC (frequency independent) to AC (frequency dependent) conductivity might be due to the change in hopping of charge carriers in PDAN and PDAN/CdSe. The total conductivity in these nanocomposites follows the Jonscher's law,

$\sigma_{\mathrm{T}}=\sigma_{\mathrm{dc}}(0)+\sigma_{\mathrm{ac}}(\omega)$

It is clear from the plot that the electrical conductivity for native PDAN and PDAN/CdSe(S) nanocomposites are as per the Almond-West universal power law given as, 


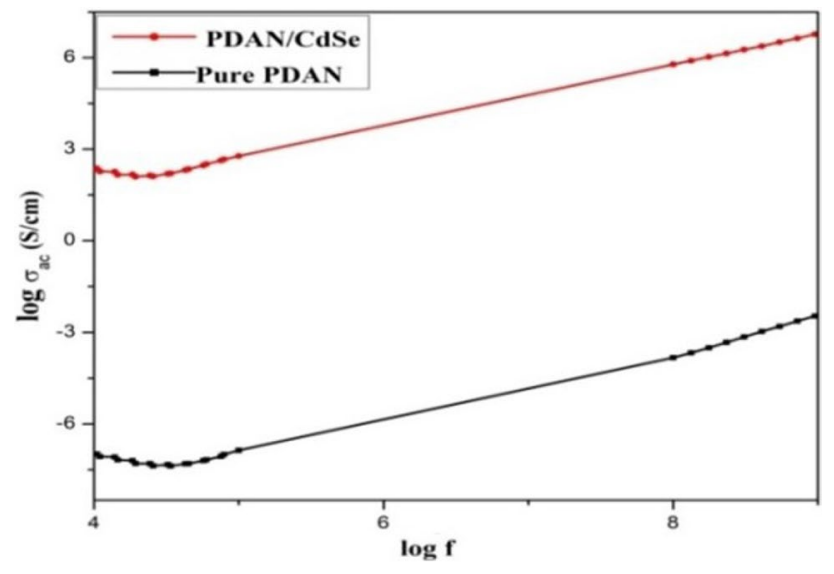

Fig. 7 Frequency dependent AC conductivity of native PDAN and PDAN/CdSe nanocomposites

$\sigma_{\mathrm{ac}}(\omega)=A \omega^{\mathrm{n}}$

where ' $A$ ' is constant, which has constant value at room temperature.' $n$ ' is an exponent which is frequency dependent. Tyagi and Shrma [10] have also used the same law for conductivity measurements of CdSe loaded poly(diallyl dimethyl ammonium chloride) (PDADMAC) polymer. It is reported in literature that in conducting polymer, PDAN, polarons are responsible for conduction. These get aligned with the direction of the external field through hopping. The fast hopping breaks the covalent bonds of the polymer. This hopping and breaking of covalent bonds mechanisms are responsible for an increase in electrical conduction in PDAN. As pulse repetition frequency increases, more and more hopping of polarons causes greater charge carriers and hence larger conductivity is observed. In PDAN/CdSe nanocomposites also, polarons becomes aligned with the field. The charge carriers generated due to the hopping of polarons together with the charge carriers liberated from CdSe semiconductor cause higher conductivity. The conductivity increases from $1 \times 10^{-3} \mathrm{~S} \mathrm{~cm}^{-1}$ for pure PDAN to $3 \times 10^{-3} \mathrm{~S} \mathrm{~cm}^{-1}$, when $\mathrm{CdSe}(\mathrm{S})$ is reinforced into the PDAN matrix. This can be calculated using the formula given in Eq. 13 [38-40].

$\sigma_{\mathrm{AC}}=\frac{G(\omega) * t}{A}$

At higher frequency the AC conductivity increases due to inter and intra chain hopping of charge carriers (polaron/bipolaron). The enhanced conductivity shows that these nanocomposites can be used for charge storage applications $[10,41]$.

\section{Conclusions}

To design the advanced energy storage devices such as rechargeable batteries, optical devices etc. the thermal stability and electrical conductivity play an important role. These parameters determine the use of these materials in electronic devices, charge storage devices and gas sensors devices, often subjected to industrial use. In the present research work, chemical oxidative polymerization method is used to prepare PDAN/CdSe nanocomposites. The XRD spectra and TEM analysis confirm the reinforcement of CdSe into the PDAN matrix. Moreover, this characterization further shows that the QDS of CdSe are formed in PDAN matrix. Thermal stability of PDAN/CdSe nanocomposites is better than that of the pure PDAN. The AC conductivity increases at higher frequency in both the samples due to the charge transport by hopping process. The reported results further confirm that the material chosen in the present research work can be used for various technological and commercial purposes.

Acknowledgements The characterization of all the samples was done at Raja Ramanna Centre for Advanced Technology, Indore (MP), India. The authors greatly acknowledge the facilities extended for the present work.

\section{Compliance with ethical standards}

Conflict of interest On the behalf of all authors, I have declared that no conflict of interest has received.

\section{References}

1. Isah S (2018) Advanced materials for energy storage devices. Asian J Nano Sci Mater 1(2):90

2. Abusalemhamadnabirquidri $S$, Mohiuddin MM, Ahmed MF, Husain J (2018) Thermal and sensing properties of polyaniline/ lead oxide nanocomposites. J Eng Res Appl 8(5):45

3. Juwhari HK, Abuobaid A, Zihlif AM, Elimat ZM (2017) Investigation of thermal and electrical properties for conductive polymer composites. J Electron Mater 46(10):5705

4. Bhatt R, Katare R, Bajpai AK (2014) Poly-dispersive nature of relaxation times characteristics of poly-(diamino-naphthalene) doped poly-(vinyl-alcohol) films from AC impedance analysis. Res J Chem Sci 4(8):82

5. Park H, Kwon TG, Park DS, Shim YB (2006) Electrocatalytic reduction of molecular oxygen at poly (1, 8-diaminonaphthalene) and poly(Co (II)-(1, 8-diaminonaphthalene)) coated electrodes. BullKorean Chem Soc 27(11):1763

6. Li Q, Qian Y, Cui H, Zhang Q, Tang R, Zhai J (2011) Preparation of poly(aniline-1, 8-diaminonaphthalene) and its application as adsorbent for selective removal of $\mathrm{Cr}(\mathrm{VI})$ ions. Chem Eng J 173:715

7. Li XG, Huang MR, Li SX (2004) Facile synthesis of poly(1, 8-diaminonaphthalene) microparticles with a very high silver-ion adsorbability by a chemical oxidative polymerization. Acta Mater 52:5363 
8. Yang B, Zhang J, Song Q, Ye X, Wang H (2016) Improved fluorescence and solubility properties of $\mathrm{N}$-substituted carboxyl poly(1,8-diaminonaphthalene) in the presence of europium cations. J Chem Pharm Res 8(4):407

9. Kaur R, Tripathi SK (2015) Study of conductivity switching mechanism of CdSe/PVP nanocomposite for memory device application. Microelectron Eng 133:59-65

10. Tyagi C, Shrma A (2016) Optimization of structural and dielectric properties of $\mathrm{CdSe}$ loaded poly(diallyl dimethyl ammonium chloride) polymer in a desired frequency and temperature window. J Appl Phys 119:014108

11. Abdelghany AM (2017) UV-irradiation assisted control of the structural, optical and thermal properties of PEO/PVP blended gold nanoparticles. Mater Chem Phys 201:100-112. https://doi. org/10.1016/j.matchemphys.2017.08.022

12. Morsi MA, Rajeh A, Menazea AA (2019) Nanosecond laserirradiation assisted the improvement of structural, optical and thermal properties of polyvinyl pyrrolidone/carboxymethyl cellulose blend filled with gold nanoparticles. J Mater Sci Mater Electron 30(3):2693-2705. https://doi.org/10.1007/s1085 4-018-0545-4

13. Salem A, Saion E, Al-Hada NM, Kamari HM, Shaari AH, Che Abdullah CA, Radiman S (2017) Synthesis and characterization of CdSe nanoparticles via thermal treatment technique. Results Phys 7:1556

14. Brus $L$ (1986) Electronic wave functions in semiconductor clusters: experiment and theory. J Phys Chem 90:2555

15. Verma M, Patidar D, Sharma KB, Saxena NS (2016) Phase transformations and thermal stability of CdSe quantum dots: cubic to hexagonal. J Inorg Organomet Polym 26:75

16. Wu Y, Wadia C, Ma W, Sadtler B, Alivisatos AP (2008) Synthesis and photovoltaic application of copper (I) sulfide nanocrystals. Nano Lett 8:2551

17. Gur I, Fromer NA, Geier ML, Alivisators AP (2005) Air-stable all-inorganic nanocrystal solar cells processed from solution. Science 310:462

18. Gangadharam R, Jayalakshmi V, Kalaiselvi J, Mohan S, Murugan R, Palanivel B (2003) Electronic and structural properties of zinc chalcogenides $\mathrm{ZnX}(\mathrm{X}=\mathrm{S}$, Se, Te). J Alloys Compd 359:22

19. Al-Hosiny N, Badawi A, Moussa MAA, El-Agmy R, Abdallah $S$ (2012) Characterisation of optical and thermal properties of CdSe quantum dots using photoacoustic technique. Int $J$ Nanoparticles 5(3):258

20. Badawi A (2015) Characterization of the optical and mechanical properties of CdSe QDs/PMMA nanocomposite films. J Mater Sci Mater Electron 26:3450

21. Abdelghany AM, Abdelrazek EM, Badr SI, Morsi MA (2016) Effect of gamma-irradiation on (PEO/PVP)/Au nanocomposite: materials for electrochemical and optical applications. Mater Des 97:532-543. https://doi.org/10.1016/j.matdes.2016.02.082

22. Kumar D, Chandra R (2001) Thermal behaviour of synthetic metals: polyanilines. Ind J Eng Mater Sci 8:209

23. Ansari R (1995) Thermal studies of conducting electroactive polymers. The University of Wollongong, Chemistry Department, Wollongong

24. Yao J, Zhao GL, Han G (2003) Synthesis and characterization of the thiourea-capped CdS nanoparticles. J Mater Sci Lett 22:1491-1493

25. Kale RB, Sartale SD, Chougule BK, Lokhande CD (2004) Growth and characterization of nanocrystalline CdSe thin films deposited by the successive ionic layer adsorption and reaction method. Semicond Sci Technol 19(8):980

26. Vijeth H, Yesappa L, Niranjana M, Ashokkumar SP, Devendrappa $H$ (2017) Investigation on structural, optical and electrical properties of polythiophene- $\mathrm{Al}_{2} \mathrm{O}_{3}$ composites. In: AIP Conference Proceedings, vol 1953, p 050008-1

27. Silva RA, Santos MJL, Rinaldi AW, Jarbin AJG, Oliverira MM (2007) Low coercive field and conducting nanocomposite formed by $\mathrm{Fe}_{3} \mathrm{O}^{4}$ and poly (thiophene). J Solid State Chem 180(12):3545

28. Patidar D, Jain N, Saxena NS, Sharma K, Sharma TP (2006) Electrical properties of $\mathrm{CdS}$ /polyaniline heterojunction. Braz J Phys 36(4A): 1210

29. Singh R, Bajpai AK, Shrivastava AK (2019) Structural, morphological and electrical studies of poly(1, 8-diaminonaphthalene)/ $\mathrm{CdSe}(\mathrm{S})$ nanocomposites for charge storage applications. Mater Res Express 6:045033

30. Bhatt R, Tiwari A, Bisen DS, Bajpai AK (2018) Analyzing p-type conjugated conducting poly(diaminonaphthalene) doped poly(vinyl alcohol) bulk hetrojunction film for organic solar cells. Mater Today Proc 5:1673

31. Mofokeng JP, Luyt AS, Tabi T, Kovacs J (2011) Comparison of injection moulded, natural fibre-reinforced composites with PP and PLA as matrices. J Therm Comp Mat 25(8):927

32. Huda MS, Drzal LT, Misra M, Mohanty AK, Williams K, Mielewski DF (2005) A study on biocomposites from recycled newspaper fiber and poly(lactic acid). Ind Eng Chem Res 44(15):5593

33. Yesappa L, Niranjana M, Ashok Kumar S, Vijetha H, Raghu S, Devendrappa $\mathrm{H}$ (2018) Characterization, electrical conductivity and electrochemical performance of polyaniline- $\mathrm{LiClO}_{4}-\mathrm{CuO}$ nano composite for energy storage applications. Polym Plast Technol Eng. https://doi.org/10.1080/03602559.2018.1466175

34. Lozano K, Barrera EV (2015) Nanofiber-reinforced thermoplastic composites. I. Thermoanalytical and mechanical analyses. J Appl Polym Sci 79:125-133. https://doi.org/10.1002/10974628(20010103)

35. Ansari R, Keivani MB (2006) Polyaniline conducting electroactive polymers thermal and environmental stability studies. E-J Chem 3(4):202

36. Wei Y, Hsueh KF (1989) Thermal analysis of chemically synthesized polyaniline and effects of thermal aging on conductivity. J Polym Sci A Polym Chem 27:4351

37. Akkaya T, Gulfen M, Olgun U (2013) Adsorption of rhodium (III) ions onto poly(1,8-diaminonaphthalene) chelating polymer: equilibrium, kinetic and thermodynamic study. React Func Polym 73:1589

38. Unal B, Durmus Z, Kavas H, Baykal A, Toprak M (2010) Synthesis, conductivity and dielectric characterization of salicylic acid$\mathrm{Fe}_{3} \mathrm{O}_{4}$ nanocomposite. Mater Chem Phys 123:184

39. Baykal A, Genc F, Elmal A, Sertkol M, Sozeri H (2016) $\mathrm{MnCr}_{\mathrm{x}} \mathrm{Fe}_{2-\mathrm{x}} \mathrm{O}_{4}$ nanoparticles: magnetic and microwave absorption properties. J Inorg Organomet Polym 26(1):134

40. Koteswararao J, Abhishek R, Satyanarayana SV, Madhu GM, Venkatesham V (2016) Influence of cadmium sulfide nanoparticles on structural and electrical properties of polyvinyl alcohol films. Express Polym Lett 10(11):883

41. Ahmed RM (2017) Polymer nanocomposite dielectric and electrical properties with quantum dots nanofiller. Mod Phys Lett $B$ 31:1750278. https://doi.org/10.1142/S0217984917502785

Publisher's Note Springer Nature remains neutral with regard to jurisdictional claims in published maps and institutional affiliations. 